\title{
Extracellular Levels of 5HT and 5HIAA Increase after an Inflammatory Process in the Rat's Insular Cortex
}

\author{
Ulises Coffeen*, Ana Canseco-Alba, Karina Simón-Arceo, Francisco Mercado, \\ Angélica Almanza, Orlando Jaimes, Francisco Pellicer \\ Instituto Nacional de Psiquiatría Ramón de la Fuente Muñiz, Mexico City, Mexico \\ Email: *coffeen@imp.edu.mx
}

Received 5 November 2015; accepted 11 February 2016; published 14 February 2016

Copyright (C) 2016 by authors and Scientific Research Publishing Inc.

This work is licensed under the Creative Commons Attribution International License (CC BY). http://creativecommons.org/licenses/by/4.0/

(c) (i) Open Access

\begin{abstract}
Serotonin (5HT) in the central nervous system has been associated with pain processing and modulation. The insular cortex (IC) plays an important role in the development and perception of the inflammatory and chronic pain. The role of the serotoninergic system in IC has not been completely studied. We used micro-dialysis in freely moving rats to determine the extracellular release of 5HT and its main metabolite (5HIAA) in the IC during an inflammatory process. Results showed an increase of extracellular levels of 5HT and 5HIAA in the IC during carrageenan-induced inflammation and this augmentation correlates with a decrease of behavioral mechanonociceptive response. Furthermore, the exogenous administration of 5HT and 5HIAA in the IC increases the nociceptive response. Our current data imply that the serotoninergic system in the IC participates in the long-term pain process.
\end{abstract}

\section{Keywords}

Pain, Inflammation, Insular Cortex, 5HT, 5HIAA

\section{Introduction}

The role of monoamines in the inflammatory and neuropathic pain has been widely described [1] [2]. In this way, serotonin (5-hydroxtryptamine [5HT]), has a differential role according to its localization and the receptors subtype in which exerts its effects. In the periphery, 5HT is a component of the so called "inflammatory soup", in which, along with other mediators such as histamine, prostaglandins, bradykinin, etc., contributes to inflamma-

*Corresponding author.

How to cite this paper: Coffeen, U., Canseco-Alba, A., Simón-Arceo, K., Mercado, F., Almanza, A., Jaimes, O. and Pellicer, F. (2016) Extracellular Levels of 5HT and 5HIAA Increase after an Inflammatory Process in the Rat's Insular Cortex. World Journal of Neuroscience, 6, 23-31. http://dx.doi.org/10.4236/wjns.2016.61003 
tory pain [2] [3].

In the central nervous system (CNS) the 5HT participation is more complex. It has been reported that the intrathecal administration of a low dose of 5HT induces antinociceptive effects in the formalin test, while a high dose induces the pronociceptive effects. This effect seems to be related to the 5-HT1A activation [4]. At this level, a low frequency of stimulation $(4 \mathrm{~Hz})$ is capable of increasing the release of $5 \mathrm{HT}$ in rats with a joint inflammatory model, causing an antinociceptive effect [5].

Very little is known about the pathophysiological relevance of the monoaminergic system in supraspinal structures related to the pain matrix. At supraspinal level, the 5HT and its main metabolite 5-HIAA levels are increased, both in the periaqueductal grey matter and in the trigeminal nucleus after a peripheral nerve axotomy, on the contrary the basal levels of 5HT decrease in the ventromedial thalamus in a neuropathic pain model induced by the ligature of the L5 and L6 spinal roots [6] [7]. In the medial prefrontal cortex, the extracellular levels of 5-hydroxyindoleacetic acid (5HIAA) and DOPAC (metabolite of dopamine) are decreased in the formalin test when there's a novel stimuli [8].

In this context, one of the cortical nuclei mainly related with the development and perception of the inflammatory and neuropathic pain is the insular cortex (IC) [9]-[13]. This nucleus acts as a pronociceptive site, since if it is selective injured the expression of pain associated behaviors decreased in animals [14]. Even more the stimulation of inhibitory dopaminergic receptors decreased the nociceptive behavior too, while the stimulation of excitatory systems, such as the glutamatergic, increases it [15]. The induction of an inflammatory process induced by an injection of carrageenan, decreases the basal levels of dopamine and increased the expression of the D2 inhibitory receptor [16].

Nevertheless, the role of serotoninergic system related to pain in this region has not been fully studied. Thus, we decide to determine if the extracellular release of 5HT and its main metabolite (5HIAA) in the IC can be modified during an inflammatory process induced by carrageenan and determine the effect of the exogenous administration of 5HT and 5HIAA directly in the rat's insular cortex after the carrageenan induced inflammation.

\section{Material and Methods}

The experiments were conducted in agreement with the ethics committee regulations of the International Association for the Study of Pain [17] and with our institution project and bioethics commission's approval.

Male Wistar rats (250 - 300 g) were raised, housed and maintained in our institution's animal house. The animals were kept in transparent acrylic individual cages, with light-dark cycles of $12: 12 \mathrm{~h}$ at $23^{\circ} \mathrm{C}$ and $52 \%$ humidity, and with ad libitum feeding and hydration.

\subsection{Inflammatory Induction and Mechanical Stimulation}

An inflammatory process was induced by the infiltration in the right hind paw of carrageenan lambda (Sigma Chemical Co. St. Louis MO, USA, CAR 1\% in saline solution, $100 \mu \mathrm{l})$.

Nociceptive tests were performed using an aesthesiometer (Von Frey mechanonociception; UgoBasile). In this test, the threshold of the flexor reflex produced by a metallic filament was determined (in grams [g]; 10 -second ramp from 0 to $50 \mathrm{~g}$ ). Four measurements from the contralateral hind paw were averaged to obtain the value for each time point (preceding $1 \mathrm{~h}$ and $3 \mathrm{~h}$ after intraplantar injection).

\subsection{HT and 5HIAA Release in the Insular Cortex}

Extracellular concentration of 5HT and its main metabolite 5HIAA were measured in the IC through micro-dialysis in freely moving rats and high performance liquid chromatography (HPLC) during the development of an inflammatory pain model.

\subsubsection{In Vivo Microdialysis}

Rats were anaesthetized with isofluorane $2 \%$ mixed with $98 \% \mathrm{O}_{2}$ and mounted in a stereotaxic frame. A guide cannula (CMA-11-Microdialysis, Acton, MA) was stereotaxically implanted into the IC. Forty eight hours after cannulation, a micro-dialysis probe (SciPro Inc. 12, $2 \mathrm{~mm}$ tip length) was inserted into the guide cannula so that its tip ended in the IC (A $=1 \mathrm{~mm}$ from bregma, $\mathrm{L}=4.8 \mathrm{~mm}, \mathrm{H}=5.8 \mathrm{~mm}$ from meninges) using coordinates according to the atlas by Paxinos and Watson (1998) [18]. Each probe was continuously perfused at $2 \mu \mathrm{l} / \mathrm{min}$ with sterile artificial cerebrospinal fluid (aCSF) (145 mMNaCl, $2.8 \mathrm{mMKCl}, 3.0 \mathrm{mM} \mathrm{CaCl}$, $5.4 \mathrm{mM}$ D-glucose, $\mathrm{pH}$ 
7.2) using a micro-infusion pump (KD Scientific, Holliston, MA, USA). Animals were individually housed for the duration of the experiment in a freely-moving system and micro-dialysate samples were collected at $20 \mathrm{~min}$ intervals into micro-vials containing $4 \mu \mathrm{l}$ of L-Glutathione $0.08 \%$ (Sigma-Aldrich) to reduce oxidation of monoamines.

\subsubsection{Biochemical Conditions (HPLC)}

An isocratic, high-performance liquid chromatography electrochemical detection (HPLC-ECD) assay was used to quantify 5HT, in 20- $\mu \mathrm{l}$ samples of micro-dialysate. A mobile phase containing $95 \%$ of $12.5 \mathrm{mM}$ citric acid, $0.07 \mathrm{mM}$ 1-octanesulfonic acid sodium salt, $0.05 \mathrm{mM}$ EDTA and $25 \mathrm{nMortho}$ phosphoric acid, and $5 \%$ of methanol HPLC (adjusted to $\mathrm{pH} 3.2$ with $\mathrm{KOH} 10 \mathrm{M}$ ), was pumped at $0.1 \mathrm{ml} / \mathrm{min}$ through an X Terra C 18 (2.1 $\AA$ $50 \mathrm{~mm}, 3.5 \mu \mathrm{m}$ ODS) column. Online data capture was performed using Waters Empower software for HPLC.

The animals were divided in the following groups: Control $(n=8)$ : basal extracellular concentration of 5HT and 5HIAA during three hours. Mechanonociception group $(n=6)$ : extracellular concentrations of 5HT and 5HIAA were measured during three hours and von frey was carried out at the beginning of the micro-dialysis, and also one and three $h$ later. Inflammation group $(n=6)$ : extracellular concentrations of 5HT and 5HIAA were measured prior to the induction of inflammation and until three hours after its induction. Mechanonociception was carried out prior to inflammation and one and three $h$ afterwards.

\subsection{Microinjection of 5HT and 5HIAA in the Insular Cortex}

Cannula implantation by stereotaxic surgery A guide cannula of $17 \mathrm{~mm}$ long (inner diameter: $21 \mathrm{G}$ ) was inserted unilaterally in the left IC (AP $=+1.0 \mathrm{~mm}$; $\mathrm{L}=+4.8 \mathrm{~mm}$; DV; -4.8 from Bregma [18]. The animals had a recovery period of $48 \mathrm{~h}$.

The day of the experiment the animals were injected through the cannula attached to a $100 \mathrm{ml}$ Hamilton syringe with either sterile saline solution, 5HT (5 $\mu \mathrm{g} / \mu \mathrm{l})$ or 5HIAA $(2.5 \mu \mathrm{g} / \mu \mathrm{l})$. The syringe was placed using a microsyringe pump (WPI, Sarasota, FL, USA). Either the drug or the vehicle was microinjected at a rate of 0.5 $\mu \mathrm{l} / \mathrm{min}$ during $2 \mathrm{~min}$. The syringe was left in place for additional $60 \mathrm{~s}$ to reduce the chance of reflux. These microinjection parameters have been reported not to induce tissular damage [19]. The dosages used in this work were chosen from previous reports demonstrating pharmacological efficacy in this range [20] [21].

\subsection{Histological Verification}

At the end of the experiment the correct micro-dialysis probe placement was verified. Briefly, the animals were intracardially perfused with physiological saline solution, followed by $10 \%$ formaldehyde. The brains were allowed to postfix for 2 days and cut in $40 \mu \mathrm{m}$ coronal slices that were immediately placed in a glass slide and digitalized in a scanner (HP Scanjet 5550C). The images were analyzed by comparing them to an anatomical atlas [18] (Figure 1).

\subsection{Statistical Analysis}

Statistically, significant differences $(p<0.05)$ between groups on extracellular concentrations of $5 \mathrm{HT}$ and 5HIAA in the IC, were established by repeated measures ANOVA, with a post hoc Tukey test. In order to establish differences in PWT between the control and each experimental group a Student's t-test was performed. Significance was considered with a value of $p<0.05$. A Pearson's correlation was performed in order to test if there was a relationship between 5HT and 5HIAA release and PWT.

\section{Results}

We measured extracellular 5HT and 5HIAA release in the IC during an inflammatory process induced by the intraplantar injection of carrageenan. Simultaneously, the behavioral mechanonociceptive response (paw withdrawal threshold, PWT, to mechanonociceptive stimulation) was measured prior to inflammation, as well as one and three hours afterwards.

The concentration of 5HT and 5HTIAA in the inflammation group was increased when compared to control and mechanonociception groups. This increase was of $23.6 \% 1 \mathrm{~h}$ after the induction of inflammation with carrageenan $(p<0.05)$ and 52\% after 3 h for 5HT $(p<0.05)$ from basal values (Figure 2(a)). 5HIAA increased progressively until $70 \%$ at $3 \mathrm{~h}(p<0.001)(p<0.05$; Figure 2(b)). 


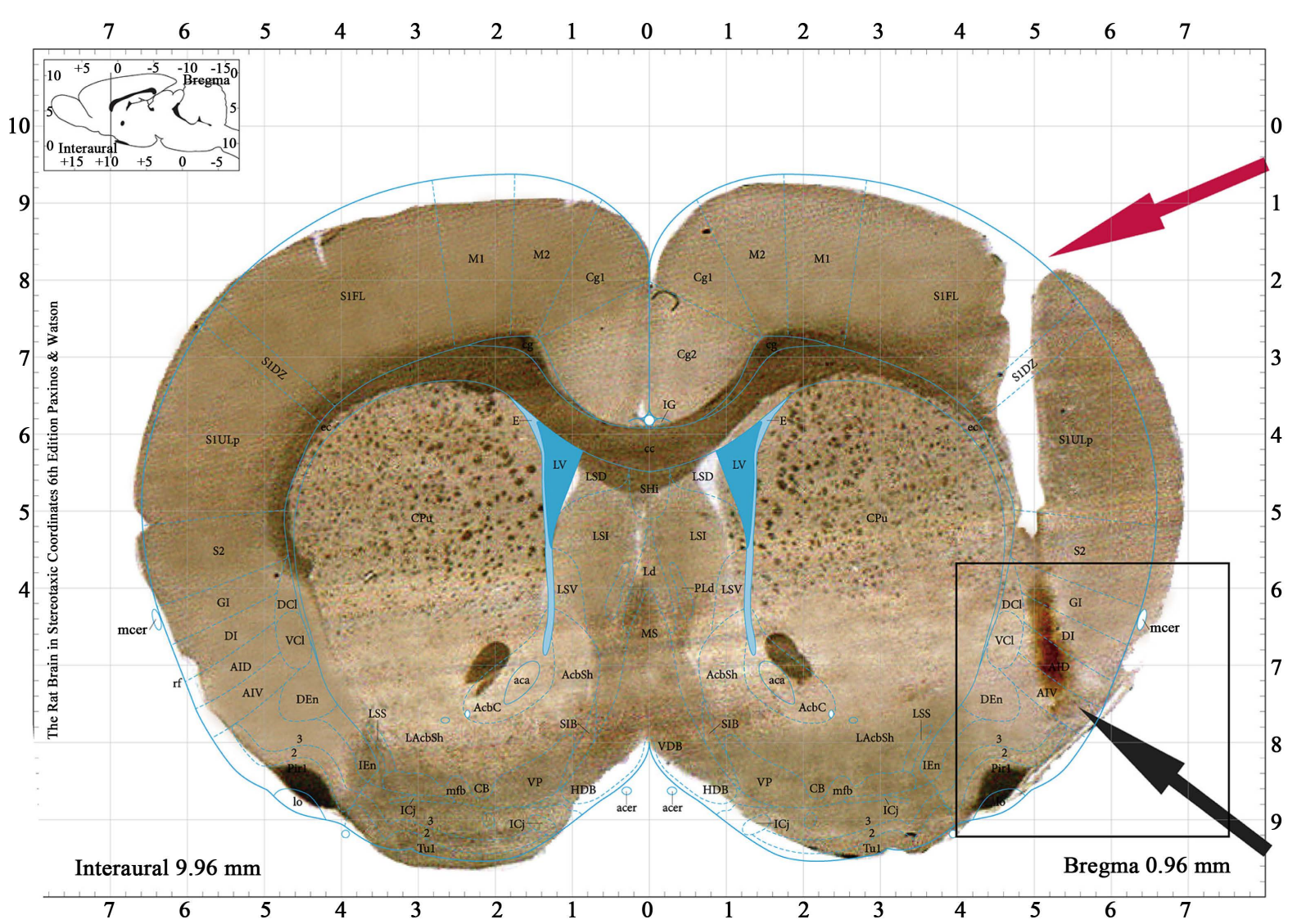

Figure 1. Histological verification of the microdialysis cannula, The figures show the guide cannula (red arrow) and membrane trace (black arrow). The membrane location (black arrow) involves the three regions of the insular cortex (granular, GI; dysgranular, DI; and agranular, AI). The animals, where off-site lesions were found, were dropped out from the " $n$ " in the corresponding group.

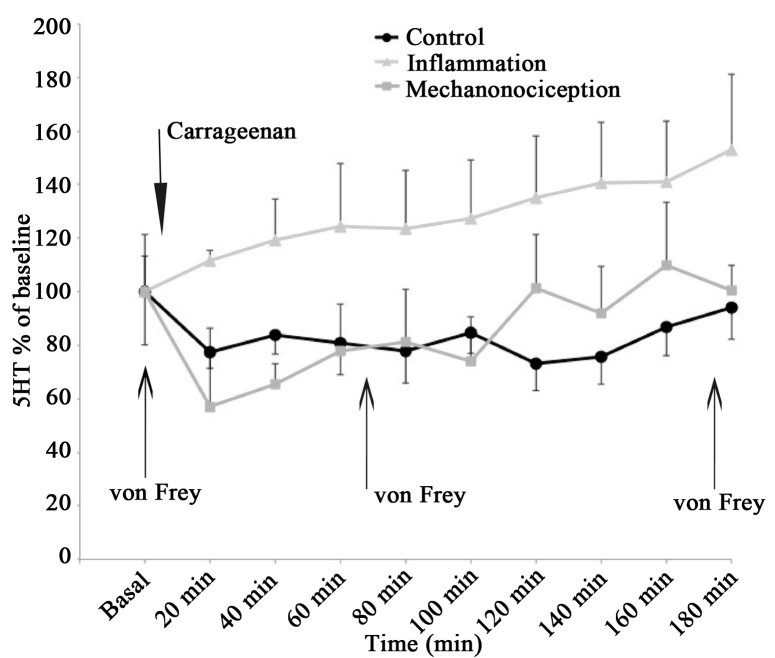

(a)

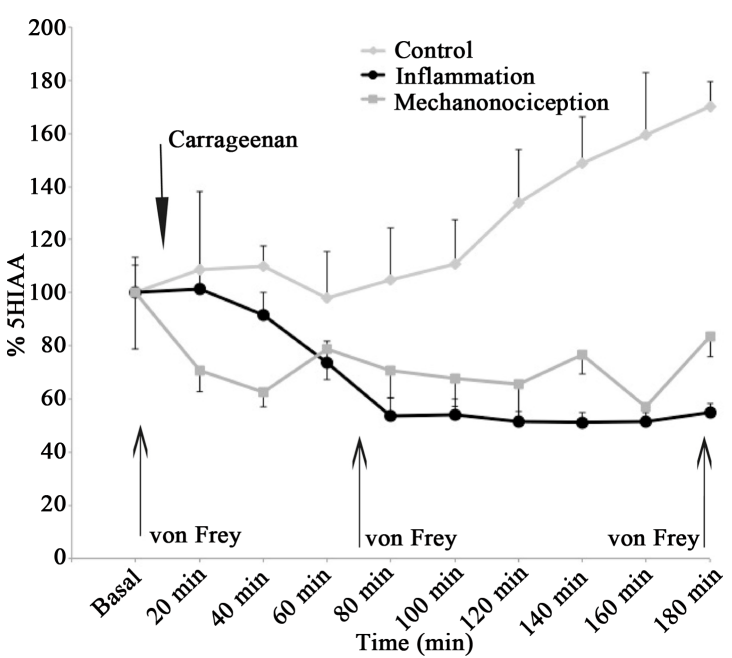

(b)

Figure 2. The graphs show extracellular levels of 5HT (a) and its main metabolite 5HIAA (b) in the insular cortex. The data is expressed as the percentage of their respective basal levels. Notice that the group with inflammation (which received an intraplantar injection of carrageenan) shows an increased percentage in the levels of 5HT and 5HIAA when compared with the control and mechanonociception groups. Repeated measures ANOVA, 5HT (F=3.794; $p=0.001, \mathrm{n}=6)$ and 5HIAA (F $=11.520 ; p=0.001, \mathrm{n}=8$ ). 
In regard of behavioral test, there was a $45 \%$ decrease of PWT at $1 \mathrm{~h}(\mathrm{t}=7.027, P=0.001)$ and $69.6 \%$ at $3 \mathrm{~h}$ $(\mathrm{t}=8.570, P<0.001)$ after inflammation in the inflammation group compared with control and mechanonociception groups (Figure 3).

Moreover, there was a negative correlation between the increase in 5HIAA release in the IC with the decrease in PWT in the inflammation group (Pearson correlation, $r=0.599, p<0.05$, Figure 4).

The intra-insular administration of the dose of $5 \mu \mathrm{g} / \mu \mathrm{l}$ induce a significantly decrease of PWT one hour after the administration of carrageenan, the administration of the metabolite 5HIAA not induce changes in PWT at this time. Three hours after the induction of the inflammatory process both groups, those injected with 5HT and 5HIAA, showed a decrease of the PWT, compared with those treated with vehicle (Figure 5).

\section{Discussion}

This study remarks the role of serotoninergic system in the insular cortex during a peripheral inflammatory process. The results show that both the extracellular level of serotonin (5HT) and its major metabolite the 5-hydroxyindoleacetic acid (5-HIAA) significantly increased compared to their physiological parameters. This correlates with a decrease of the paw withdrawal threshold. Interestingly, the repeated acute mechanical stimulation doesn't modify the release of 5HT or its metabolite. This suggests that the serotoninergic system in the insular cortex has an important role in the long-term pain process (modulated by supraspinal structures) and not in the acute process (modulated mainly by the spinal cord).

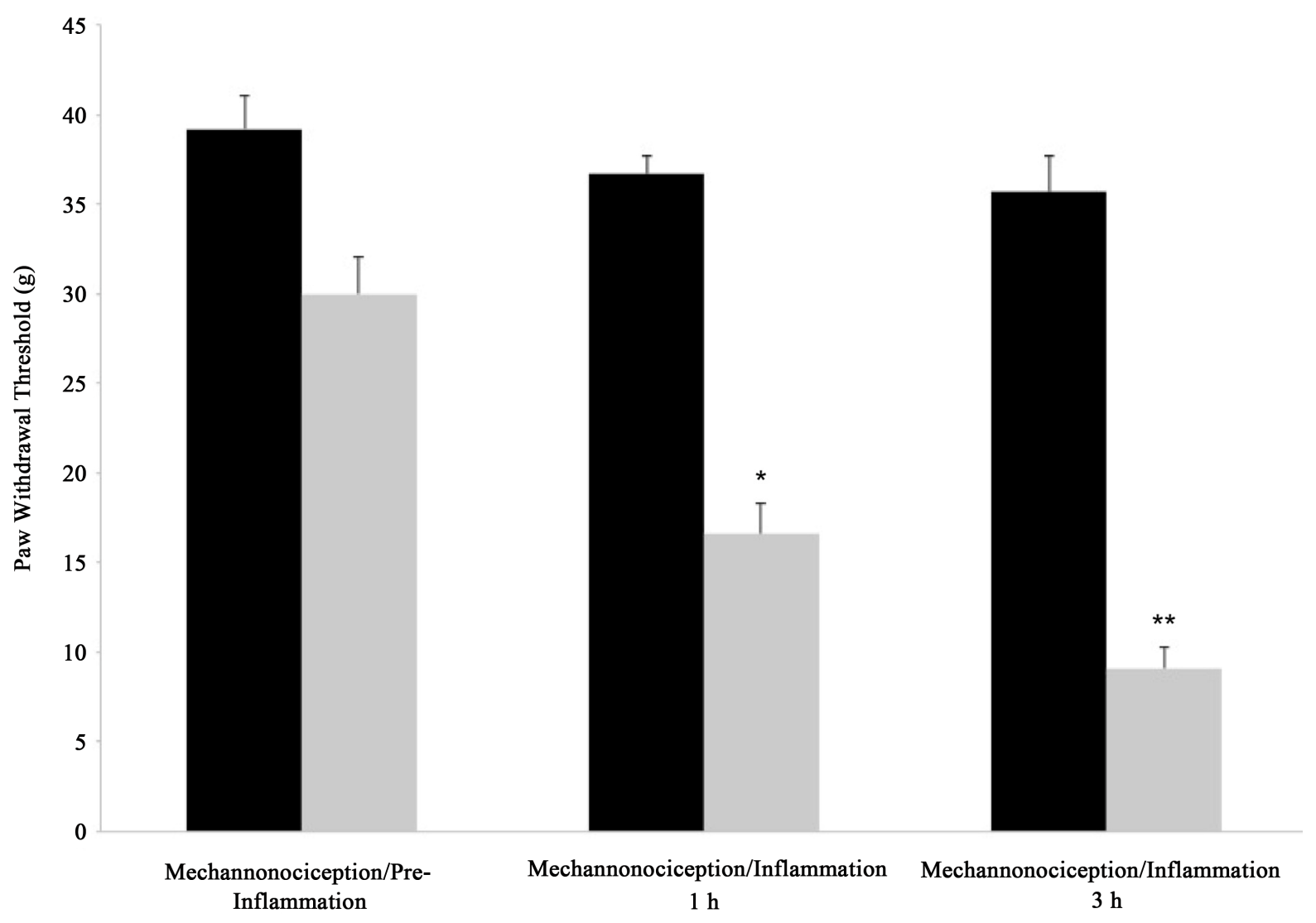

Figure 3. Antialgesic nociceptive response in the inflammation and mechanonociception groups measured as paw withdrawal threshold (PWT) in g after a mechanonociceptive stimulus. The figure shows PWT before the induction of inflammation with carrageenan $1 \%(100 \mu \mathrm{l})$ in the inflammation group in the first set of bars. The second and third sets of bars show PWT $1 \mathrm{~h}$ and $3 \mathrm{~h}$ after inflammation, respectively. The mechanonociception groups did not have inflammation induced and were tested in parallel with the inflammation group. Notice that the inflammation group has no difference in PWT before inflammation compared to the mechanonociception group and shows a progressively decreased PWT $1 \mathrm{~h}\left({ }^{*} \mathrm{t}=9.698, p=0.001\right)$ and $\left.3 \mathrm{~h}^{(* * *} \mathrm{t}=7.852, p<0.001\right)$ thereafter. 


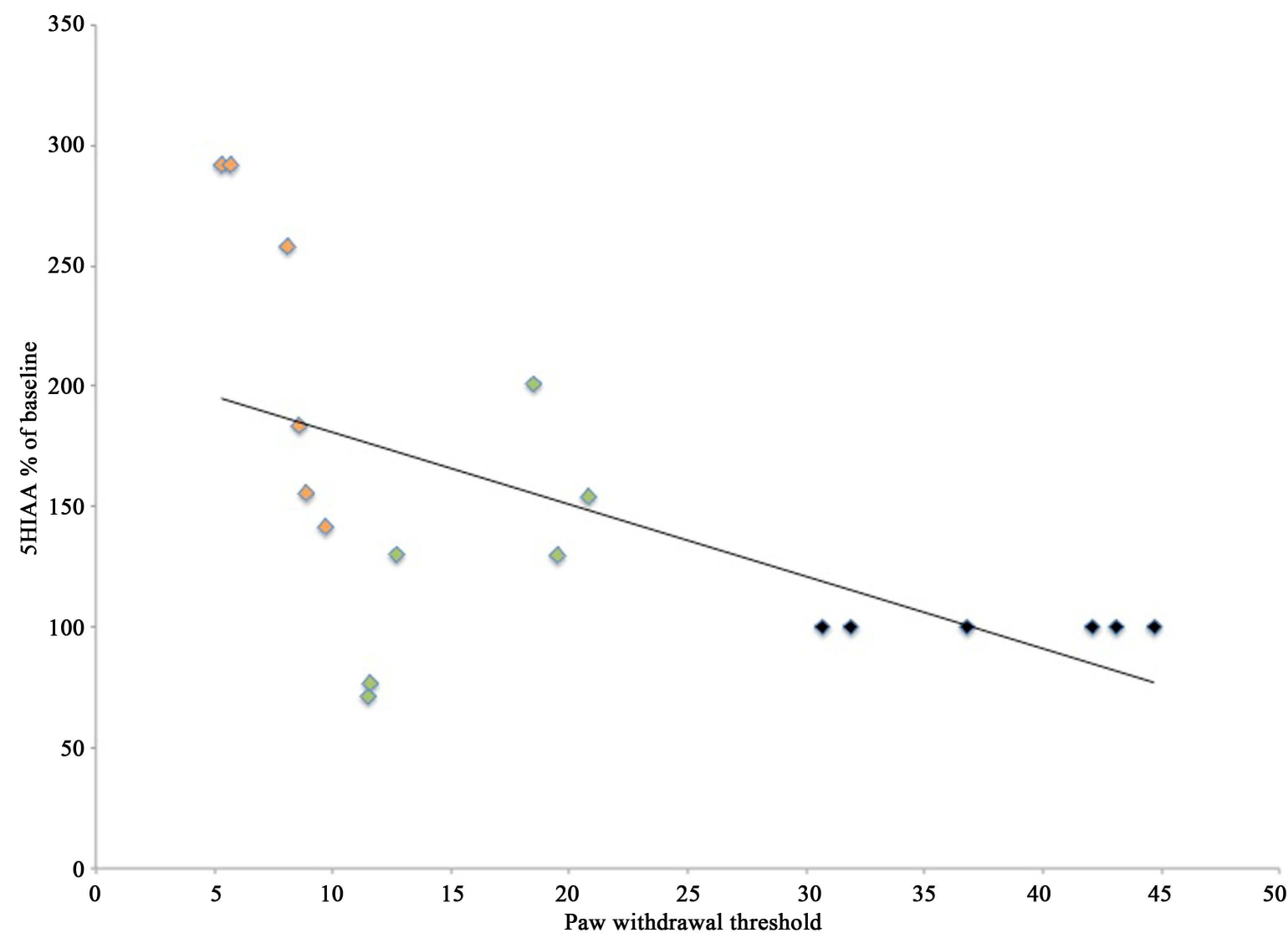

Figure 4. The figure represents a negative correlation between paw withdrawal threshold (PWT) and extracelular levels of 5HIAA. The dots in orange depict the measurements three hours after the carrageenan injection, the green ones $1 \mathrm{~h}$ afterwards and the black ones show the status prior to inflammation. Notice that as the release of 5HIAA increases, the PWT decreases and vice versa (Pearson correlation, $\mathrm{r}=0.599, p<0.05$ ).

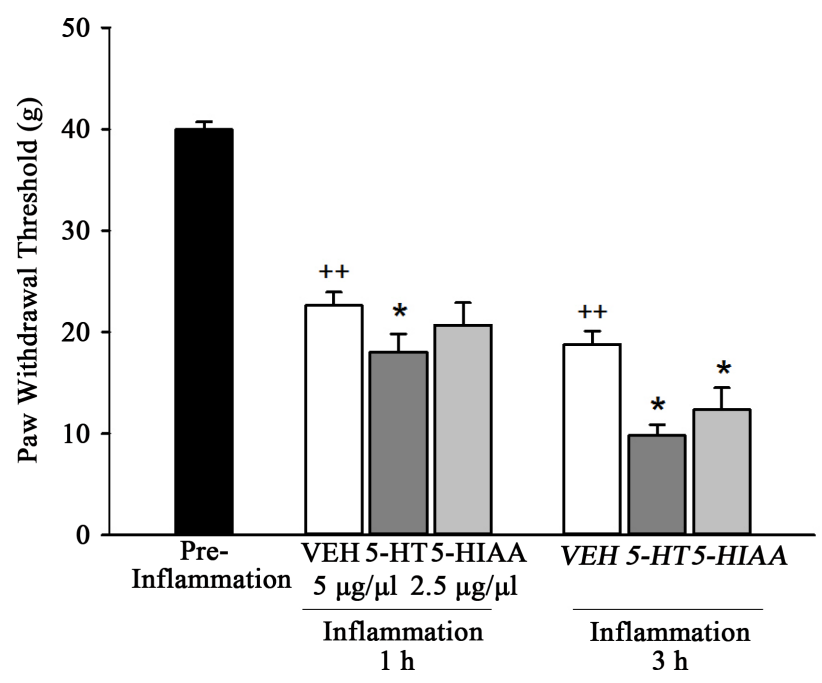

Figure 5. The figure shows the paw withdrawal threshold (PWT) in g after a mechanonociceptive stimulus in response of the intra-insular administration of 5HT, 5HIAA or vehicle after 1 and 3 hours after the inflammation with carrageenan. The dark bar represent the basal pre-inflammation levels of PWT. The white bars represent the PWT of the vehicle treated group. The dark gray is the group treated with 5HT and the light grey the 5HIAA group (U the Mann Whitney ${ }^{*} p<0.05$, experimental vs. vehicle comparisons and ${ }^{++} p<0.01$, vehicle vs. pre-inflammatory group). 
These results are in agreement to those reported by Zhang et al., they observed that extracellular concentrations of 5HT and 5-HIAA in spinal dorsal horn and periaqueductal gray (PAG) significantly increased following carrageenan inflammation [22]. Moreover as seen above, the 5HT and 5HIAA levels increase after a peripheral nerve axotomy in the periaqueductal grey matter and in the trigeminal nucleus. However, 5HT and 5HIAA levels, decrease in ventromedial thalamus and medial prefrontal cortex in a neuropathic and in formalin test respectively [6]-[8]. This discrepancy on these results may be due to the brain region studied, the pain model used or even the technique employed for the measurement of the release of the neurotransmitter. We used micro-dialysis in freely moving rats, this technique allows punctual and physiological measurement of the neurotransmitter, while the animal is behavioral free. Moreover we can record in parallel the nociceptive response, while in previous articles brain homogenates were used, in which the brain activity is indirectly measured.

Furthermore, the administration of 5HT and 5HIAA directly in the insular cortex increases the nociceptive response one and three hour after the induction of an inflammatory process, which means that the augmentation of this neurotransmitter in the IC promotes nociceptive responses in the rat. As far as we know there aren't another study with this experimental approach, so these primary results, together with the increase of the extracellular level of serotonin 5HT and 5-HIAA in the CI allow us to relate the serotoninergic system with long-term inflammatory pain.

One of the most remarkable results of this study is the important increase of the extracellular levels of the metabolite 5HIAA (up to 70\% respect the basal values, Figure 2) during the inflammatory process. Even more, there is a correlation between this augmentation and the increase of the behavioral responses associated with the pain process. It has been recently reported that this metabolite is responsible of phenomenon like the thermal hyperalgesia in the spinal cord or sciatic nerve [23]. Since the levels of 5HIAA increase with the carrageenaninduced inflammation in the IC, this work corroborates that the IC acts as a pronociceptive site in medium and long-term processes, in this case through the serotoninergic system.

The IC receives serotonergic innervation [24] and the major serotonin receptor subtypes expressed in this region are 5-HT1A and 5-HT2A [25] and also the ion channel receptor 5-HT3 [26]. On this basis it may be thought that pharmacological manipulations with agonist and antagonist may exert a differential role in pain modulation in the IC, acting both in pyramidal and gabaergic interneuron in this brain region.

The complex role of the actions of 5HT could be related to the existence of multiple 5HT receptors expressed both at the periphery and in the central nervous system. Nevertheless, the effect of 5HT not only depends on the receptor subtype mediating its action but also on the physiological/pathophysiological status of the animal. For example, in healthy animals, spinal 5HT apparently exerts mainly an inhibitory influence of pain signaling mechanisms whereas in animals sensitized by lesion of the peripheral and/or the central nervous system, the bulbo-spinal 5HT system may exacerbate pain [27]. In this regard, the extracellular levels of 5HT and 5HIAA in the IC do not vary in the acute pain process, but increase during an inflammatory process.

\section{Conclusion}

Despite being a preliminary study, our data point out that the serotoninergic system plays a pronociceptive role in the IC, which is one of the most important structures for the development and maintenance of inflammatory and chronic pain at central level. Further studies must attempt to elucidate which 5HT receptor is implicated in the nociceptive process in the insular cortex mediated by 5HT and 5HIAA.

\section{Acknowledgements}

This project was partially supported by the CONACyT grant 167134 and INPRF NC13167134 to UC.

\section{References}

[1] Pellicer, F., Ortega-Legaspi, J., López-Avila, A., Coffeen, U. and Jaimes, O. (2010) Dopamine Pathways and Receptors in Nociception and Pain. Pharmacology of Pain, IASP Press, 241-253.

[2] Sommer, C. (2004) Serotonin in Pain and Analgesia. Molecular Neurobiology, 30, 117-125. http://dx.doi.org/10.1385/MN:30:2:117

[3] Viguier, F., Michot, B., Hamon, M. and Bourgoin, S. (2013) Multiple Roles of Serotonin in Pain Control Mechanisms-Implications of 5-HT 7 and Other 5-HT Receptor Types. European Journal of Pharmacology, 716, 8-16. http://dx.doi.org/10.1016/j.ejphar.2013.01.074 
[4] Oyama, T., Ueda, M., Kuraishi, Y., Akaike, A. and Satoh, M. (1996) Dual Effect of Serotonin on Formalin-Induced Nociception in the Rat Spinal Cord. Neuroscience Research, 25, 129-135. http://dx.doi.org/10.1016/0168-0102(96)01034-6

[5] Sluka, K., Lisi, T. and Westlund, K. (2006) Increased Release of Serotonin in the Spinal Cord During Low, But Not High, Frequency Transcutaneous Electric Nerve Stimulation in Rats with Joint Inflammation. Archives of Physical Medicine and Rehabilitation, 87, 1137-1140. http://dx.doi.org/10.1016/j.apmr.2006.04.023

[6] Colado, M., Del Rio, J. and Peralta, E. (1994) Neonatal Guanethidine Sympathectomy Suppresses Autotomy and Prevents Changes in Spinal and Supraspinal Monoamine Levels Induced by Peripheral. Pain, 56, 3-8. http://dx.doi.org/10.1016/0304-3959(94)90144-9

[7] Goettl, V., Huang, Y., Hackshaw, K. and Stephens, R. (2002) Reduced Basal Release of Serotonin from the Ventrobasal Thalamus of the Rat in a Model of Neuropathic Pain. Pain, 99, 359-366. http://dx.doi.org/10.1016/S0304-3959(02)00209-9

[8] Ford, G. and Moriarty, O. (2008) Investigating the Effects of Distracting Stimuli on Nociceptive Behaviour and Associated Alterations in Brain Monoamines in Rats. European Journal of Pain, 12, 970-979. http://dx.doi.org/10.1016/j.ejpain.2008.01.002

[9] Ohara, P., Vit, J. and Jasmin, L. (2005) Cortical Modulation of Pain. Cellular and Molecular Life Sciences CMLS, 62, 44-52. http://dx.doi.org/10.1007/s00018-004-4283-9

[10] Isnard, J., Magnin, M., Jung, J., Maguiere, F. and Garcia-Larrea, L. (2011) Does the Insula Tell Our Brain That We Are in Pain? Pain, 152, 946-951. http://dx.doi.org/10.1016/j.pain.2010.12.025

[11] Lorenz, J. and Casey, K. (2005) Imaging of Acute versus Pathological Pain in Humans. European Journal of Pain, 9, 163-165. http://dx.doi.org/10.1016/j.ejpain.2004.07.009

[12] Brooks, J. and Tracey, I. (2007) The Insula: A Multidimensional Integration Site for Pain. Pain, 128, 1-2. http://dx.doi.org/10.1016/j.pain.2006.12.025

[13] Coffeen, U., Simon, K., Mercado, F., Almanza, A., Magis, L., Jaimes, O. and Pellicer, F. (2012) Central Processing of Neuropathic Pain: An Integrative Approach. Salud Mental, 35, 367-374.

[14] Coffeen, U., Manuel Ortega-Legaspi, J., López-Muñoz, F.J., Simón-Arceo, K., Jaimes, O. and Pellicer, F. (2011) Insular Cortex Lesion Diminishes Neuropathic and Inflammatory Pain-Like Behaviours. European Journal of Pain, 15, 132-138. http://dx.doi.org/10.1016/j.ejpain.2010.06.007

[15] Coffeen, U., López-Avila, A., Ortega-Legaspi, J.M., del Ángel, R., López-Muñoz, F.J. and Pellicer, F. (2008) Dopamine Receptors in the Anterior Insular Cortex Modulate Long-Term Nociception in the Rat. European Journal of Pain, 12, 535-543. http://dx.doi.org/10.1016/j.ejpain.2007.08.008

[16] Coffeen, U., Ortega-Legaspi, J.M., de Gortari, P., Simón-Arceo, K., Jaimes, O., Amaya, M.I. and Pellicer, F. (2010) Inflammatory Nociception Diminishes Dopamine Release and Increases Dopamine D2 Receptor mRNA in the Rat's Insular Cortex. Molecular Pain, 6, 75. http://dx.doi.org/10.1186/1744-8069-6-75

[17] Zimmermann, M. (1983) Ethical Guidelines for Investigations of Experimental Pain in Conscious Animals. Pain, 16, 109-110. http://dx.doi.org/10.1016/0304-3959(83)90201-4

[18] Paxinos, G. and Watson, C. (1998) The Rat Brain in Stereotaxic Coordinates. Academic Press, San Diego.

[19] Peterson, S. (1998) Drug Microinjection in Discrete Brain Regions. David Kopf Instruments, Tujunga, 1-6.

[20] Green, R., Gillin, J. and Wyatt, R. (1976) The Inhibitory Effect of Intraventricular Administration of Serotonin on Spontaneous Motor Activity of Rats. Psychopharmacology, 51, 81-84. http://dx.doi.org/10.1007/BF00426326

[21] Warbritton, J., Stewart, R. and Baldessarini, R. (1980) Increased Sensitivity to Intracerebroventricular Infusion of Serotonin and Deaminated Indoles after Lesioning Rat with Dihydroxytryptamine. Brain Research, 183, 355-366. http://dx.doi.org/10.1016/0006-8993(80)90470-9

[22] Zhang, Y.Q., Gao, X., Zhang, L.M. and Wu, G.C. (2000) The Release of Serotonin in Rat Spinal Dorsal Horn and Periaqueductal Gray Following Carrageenan Inflammation. Neuroreport, 11, 3539-3543. http://dx.doi.org/10.1097/00001756-200011090-00027

[23] Chen, Y., Palm, F., Lesch, K.-P., Gerlach, M., Moessner, R. and Sommer, C. (2011) 5-Hydroxyindolacetic Acid (5-HIAA), a Main Metabolite of Serotonin, Is Responsible for Complete Freund's Adjuvant-Induced Thermal Hyperalgesia in Mice. Molecular Pain, 7, 21. http://dx.doi.org/10.1186/1744-8069-7-21

[24] Linley, S.B., Hoover, W.B. and Vertes, R.P. (2013) Pattern of Distribution of Serotonergic Fibers to the Orbitomedial and Insular Cortex in the Rat. Journal of Chemical Neuroanatomy, 48-49, 29-45. http://dx.doi.org/10.1016/j.jchemneu.2012.12.006

[25] Santana, N., Bortolozzi, A., Serrats, J., Mengod, G. and Artigas, F. (2004) Expression of Serotonin1A and Serotonin2A Receptors in Pyramidal and GABAergic Neurons of the Rat Prefrontal Cortex. Cerebral Cortex, 14, 1100-1109. http://dx.doi.org/10.1093/cercor/bhh070 
[26] Tuerke, K.J., Limebeer, C.L., Fletcher, P.J. and Parker, L.A. (2012) Double Dissociation between Regulation of Conditioned Disgust and Taste Avoidance by Serotonin Availability at the 5-HT(3) Receptor in the Posterior and Anterior Insular Cortex. The Journal of Neuroscience: The Official Journal of the Society for Neuroscience, 32, 13709-13717. http://dx.doi.org/10.1523/JNEUROSCI.2042-12.2012

[27] Kayser, V., Latrémolière, A., Hamon, M. and Bourgoin, S. (2011) N-Methyl-D-Aspartate Receptor-Mediated Modulations of the Anti-Allodynic Effects of 5-HT1B/1D Receptor Stimulation in a Rat Model of Trigeminal Neuropathic Pain. European Journal of Pain, 15, 451-458. http://dx.doi.org/10.1016/j.ejpain.2010.09.012 